\title{
Frequency of diagnostic tests in practice
}

\author{
Bogdan Żółtowski ${ }^{*}$, Leonel Castaneda ${ }^{2}$ \\ ${ }^{1}$ UTH Technical and Commercial University, Jutrzenki 135 str., 02-231 Warsaw, Poland \\ ${ }^{2}$ EAFIT University, Colombia
}

\begin{abstract}
The elaboration concerns methods of determination of term of technical equipment next diagnostication. The purpose of it is presentation of three methods related with determination of time of the next examination of equipment state for needs of devices operating system according to technical state.
\end{abstract}

\section{Introduction}

During operation the machines' user, on the strength of achieved information on their technical state, creates rules of maintenance them in the state of ability.

Such strategy of operation is called as an operational strategy according to technical conditions. Indispensable elements of this strategy are methods, procedures and means of technical diagnostics, which enable realisation of three essential elements of diagnostic examinations of devices:

- examination and appreciation of devices' technical condition,

- localisation of occurred damages,

- prediction of state changes (forecasting of reliable operation time).

To carry out those examinations, the user should dispose of a set of appropriate procedures: procedure of state control DKS, of damage localisation DLU and of state forecasting.

In case of procedures of state control and damage localisation there is accessible a rich literature in form of manuals (manufacturer's editions) or in form of technological processes of diagnostics (user's trade editions).

For needs of strategy of machines operation according to the technical state, there have been worked out three different methods of determination of term of the next diagnostic examination (for their reliable operation), manner of determination of which is presented in this elaboration.

\section{The problem characteristics}

The strategy of operation according to the technical conditions is based on making operational decisions on the strength of current estimation of technical state of machines, their assemblies, or parts (fig.1).

*Corresponding author: bogdan.zoltowski@uth.edu.pl 
The current machine's technical state, modelled by values of measured state symptoms, is the basis of the operational decision. Correct realisation of this strategy requires using of efficient methods and means of the technical diagnostics as well as trained technical personnel. It also requires overcoming of decision-makers' mistrust concerning effectiveness of such manner of operation. Economic effects of such operational manner are incommensurably higher than in other strategies, what ensures success and great interest of this solution.

The basic condition of this strategy success is accessibility of simple and effective diagnostic methods, best of all incorporated in manufactured machines, which are then supervised in a system of the state monitoring.

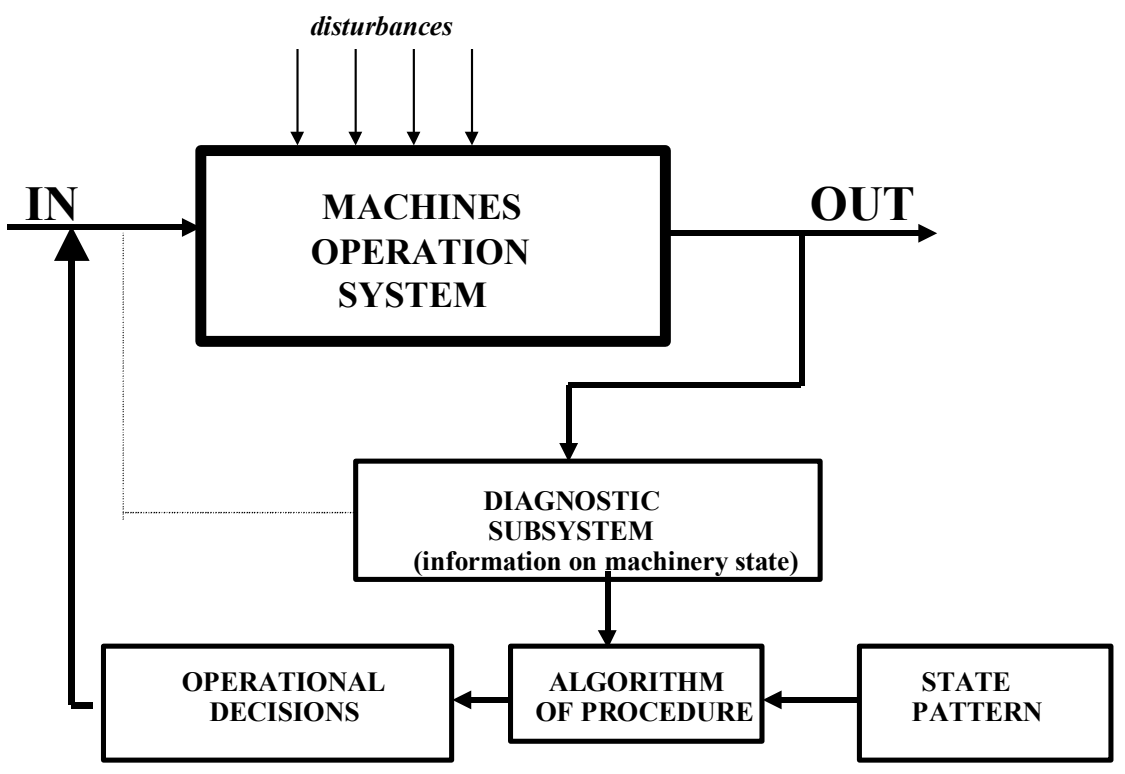

Fig.1. Diagnostic control of machines operation system

Based on known strategies of machines operation [4], in industrial practice there are built systems of machines' technical operations. Among the most spread ones we can find:

- $\quad$ system of prophylactic changes built mainly on the strength of operational strategy according to activity; (for single reliable objects - where prophylactic changes are carried out to avoid failure)

- according to a fixed plan - preventive system of technical operations; built based on strategy according to amount of performed work; (in advance planned range and frequency of technical operations, independently on current state, that is needs),

- according to a fixed plan - preventive system of technical operations with diagnostics; (like above, but helped by partial diagnostics of machine's state),

- system of technical operations according to the state; (servicing activities frequency and range - are determined based on current machine's technical state).

Some of the basic problems of technical operations system according to technical state is the need of knowledge of the way of determination of the next term of state diagnostication, determined depending on current values of measured state symptoms. 
Let phenomenon of devices' technical state destruction is represented by the time series $S_{t}=\left\langle s_{1}, s_{2}, s_{3}, \ldots, s_{b}\right\rangle$, it means by the set of discreet observations $\{\mathrm{St}=\zeta(\Theta) ; \Theta=\Theta 1$, $\Theta 2, \ldots, \Theta b\}$ of any unsteady stochastic process $\zeta(\Theta)$. With assumption, that mechanism of changes of stochastic process values in time $t \in(\Theta 1, \Theta b)$ is shaped by the trend $\mu(\Theta)$ disturbed by various random interactions $\eta(\Theta)$ :

where:

$$
\mathrm{yt}=\mu(\Theta)+\eta(\Theta)
$$

$\mu(\Theta)$ - characterises a determined component of the time series yt; it describes a developmental tendency of observed diagnostic parameter $S(\Theta)$,

$\eta(\Theta)$ - characterises aberrations from the trend and expresses effects of accidental parameters (ground and climate conditions, quality of maintenance);

there is also constructed an estimation $\{\mu p(\Theta) ; t=1,2, \ldots, b\}$ for unknown form of the trend $\mu(\Theta)$, which would ensure an appropriate accuracy of forecast $\operatorname{Sp}(\mathrm{t})$, with extrapolation $\mu p(\Theta)$ for time interval of devices usage (mileage) $(\Theta b, \Theta p)$, where: $\Theta p=\Theta b+\tau$.

As permissible period of usage (time of reliable operation) there is accepted the time, in which limits of error interval for individual forecast:

$$
\sigma(\mathrm{S} \mathrm{t}, \mathrm{S} \mathrm{p}, \mathrm{P}(\mathrm{St}, \tau))
$$

determined on a subset $\Omega y \subset \Omega$ of accessible realisations of observed parameters $\{\operatorname{Sj}(\Theta)\}$ as well as of forecasts of them $\{\mathrm{S} \mathrm{j}, \mathrm{p}\}$ according to accepted predictor $\mathrm{P}(\mathrm{St}, \tau)$, do not exceed limiting values $\{\mathrm{Sj}, \mathrm{gr}\}$.

Value of permissible time of device's reliable operation determines the temporal horizon of a forecast $\tau \mathrm{j} 0[1,2]$ :

a) for which there will not be observed any exceeding of limiting value of diagnostic parameter $\mathrm{Sj}$,gr by the limit of the forecast error interval determined by the radius $\mathrm{r} \sigma$ (method of forecast error value levelling):

where:

$$
\mathrm{r} \sigma=\mathrm{q} \sigma \mathrm{p}
$$

q - is a stable parameter calculated from the student's distribution table for required confidence level $\gamma$ and K-2 numbers of degree of freedom, $\sigma p$ - standard deviation of a random component of forecast error ep.

b) for which there will not be observed any exceeding of limiting value of diagnostic parameter Sjgr by forecasting value of diagnostic parameters (method of diagnostic parameter limiting value levelling),

c) for which there will not be observed any exceeding of limiting value of diagnostic parameter Sjgr by measured value of diagnostic parameter (method of diagnostic parameter change estimation).

\section{Method of determination of reliable operation time by means of forecast error value levelling}

In case of strategy of operation according to technical state, a required form of the devices' state forecast PST is the term of the next diagnostication $\Theta_{\mathrm{b} 1}$ :

$$
\operatorname{PST}=<\Theta_{b 1},>
$$

As the value $\Theta_{b 1}$ it is proposed to accept a value of permissible device reliable operation time, determined by a value of horizon $\tau_{j}{ }^{0}$, fixed as a point of intersection of a line of diagnostic parameter limiting value $\mathrm{Sj}$,gr with the bottom (with assumption that $\mathrm{Sj}(\Theta b)>\mathrm{Sj}$,gr) or upper 
(with assumption that $\mathrm{Sj}(\Theta \mathrm{b})<\mathrm{Sj}$,gr) limit of forecast error interval (fig.2) determined by radius $r \sigma$ for two confidence coefficients:

a) r $\sigma 0,01$ for confidence level $1-\gamma=0,99$, what responds to probability of value $p=0,01$, where in the interval determined by a horizon $\tau j^{*}$ a diagnostic parameter $\mathrm{Sj}$ achieves a limiting value Sjgr;

b) $r \sigma 0,05$ for confidence level $1-\gamma=0,95$, what responds to probability of value $p=0,05$, where in the interval determined by a horizon $\tau \mathrm{j}^{* *}$ a diagnostic parameter $\mathrm{Sj}$ achieves a limiting value Sjgr.

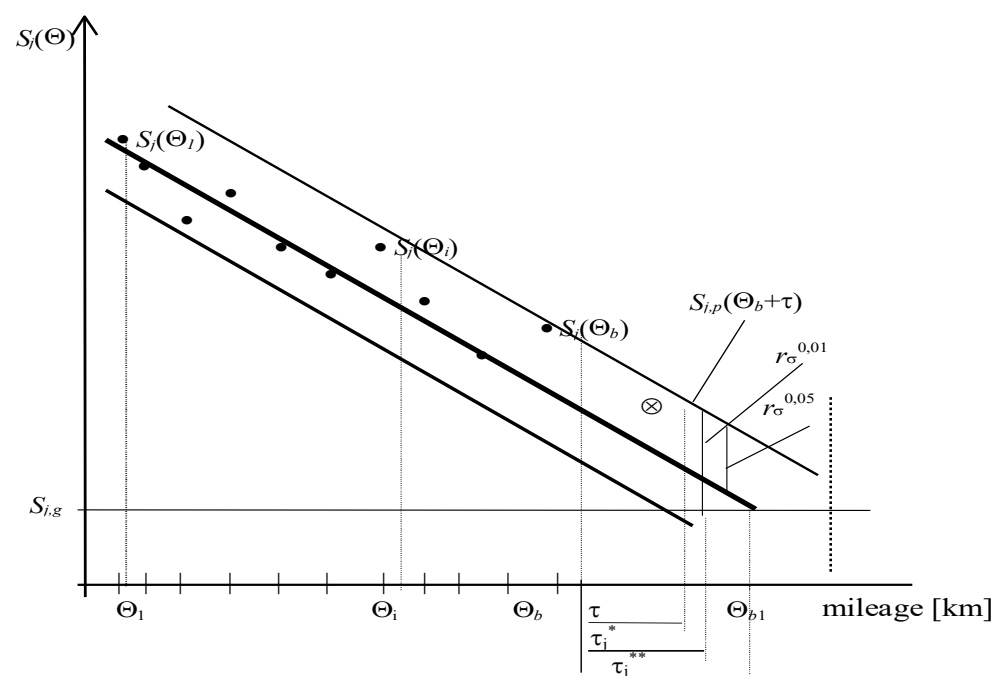

Fig.2. Determination of the term of the next devices diagnostication $\Theta b 1$ by means of the method of forecast error value levelling for $\mathrm{Sj}(\Theta \mathrm{b})>\mathrm{Sj}$,gr. $[\Theta 1$ - the beginning of device operation, $\Theta \mathrm{b}$ - the last device diagnostication, $\Theta b 1$ - the term of the next device diagnostication].

Three options are then distinguished:

a) not exceeding by a controlled diagnostic parameter of a limit determined by a radius r $\sigma 0,01$ is interpreted as a lack of alarm signal for careful and more exact diagnostic observation of the device;

b) exceeding by a controlled diagnostic parameter of a limit determined by a radius r $\sigma 0,01$ is interpreted as an alarm signal for careful and more exact diagnostic observation of the device (alert threshold);

c) moment of exceeding by a controlled diagnostic parameter of a limit determined by a radius $r \sigma 0,05$ is interpreted as the time $\Theta b 1$ - the term of the next device diagnostication (alarm threshold).

Values $\mathrm{Sj}, \mathrm{p}(\Theta \mathrm{b}+\tau)$ and $\Theta b 1$, with assumption that the Brown-Mayer's method of the first order is an optimal method of forecasting, are calculated according to expressions:

where:

$$
\mathrm{Sj}, \mathrm{p}(\Theta b+\tau)=\operatorname{at}(0)+\tau \operatorname{at}(1)
$$

and:

$$
\begin{gathered}
\operatorname{at}(0)=\operatorname{Mt}=2 \operatorname{Mt}(1)-\operatorname{Mt}(2) \\
\operatorname{at}(1)=\mathrm{Tt}=\frac{\alpha}{(1-\alpha)}(\operatorname{Mt}(1)-\operatorname{Mt}(2))
\end{gathered}
$$

$$
\operatorname{Mt}(1)=\alpha y t+(1-\alpha) \operatorname{Mt}-1(1)
$$




$$
\begin{gathered}
\operatorname{Mt}(2)=\alpha \operatorname{Mt}(1)+(1-\alpha) \operatorname{Mt}-1(2) \\
\alpha \text { - parameter of exponential smoothing, } \alpha \in(0,1) \\
\Theta \mathrm{b}_{1}=\Theta \mathrm{b}+\frac{\tau\left(\mathrm{S}_{j}\left(\Theta_{b}\right)-r_{\sigma}\right)}{S_{j}\left(\Theta_{b}\right)-S_{j, p}\left(\Theta_{b}+\tau\right)}
\end{gathered}
$$

The time interval $(\Theta 1, \Theta b)$ will then be a period of estimation of forecast error value ep and radius $r \sigma$, whilst the time interval after $\Theta b$ will be a period of active forecasting, i.e.: determination of forecasting value of diagnostic parameter after time $\tau, \operatorname{Sj}, p(\Theta b+\tau)$;

b) determination of a radius $r \sigma(\Theta b+\tau)$ value;

c) determination of the term of the next device diagnostication $\Theta_{\mathrm{b} 1}$.

\section{Method of determination of the time of reliable operation by means of levelling of diagnostic parameter limiting value}

In this method as a term of the next device diagnostication $\Theta b 1$ is proposed to accept a value of permissible time of device reliable operation, determined by a horizon value $\tau \mathrm{j} 0$, fixed as a point of intersection of lines (fig.3) of diagnostic parameter value $\mathrm{Sj}$ with:

a) lower (with assumption, that $\mathrm{Sj}(\Theta \mathrm{b})>\mathrm{Sj}$,gr) limit for two levels of limiting values:

$$
\begin{aligned}
& S_{j, g r}{ }^{*}=\frac{2}{10}\left|S\left(\Theta_{1}\right)-S_{j, g r}\right|+S_{j, g r} \\
& \quad S_{j, g r}{ }^{* *}=\frac{1}{10}\left|S\left(\Theta_{1}\right)-S_{j, g r}\right|+S_{j, g r}
\end{aligned}
$$

b) or upper (with assumption, that $\mathrm{Sj}(\Theta b)<\mathrm{Sj}, \mathrm{gr}$ ) limit determined for two levels of limiting values:

$$
\begin{aligned}
& \mathrm{Sj}, \mathrm{gr}^{*}=\mathrm{Sj}, \mathrm{gr}-\frac{2}{10}\left|S\left(\Theta_{1}\right)-S_{j, g r}\right| \\
& \mathrm{Sj}, \mathrm{gr}^{* *}=\mathrm{Sj}, \mathrm{gr}-\frac{1}{10} \quad\left|S\left(\Theta_{1}\right)-S_{j, g r}\right|
\end{aligned}
$$

Values $\mathrm{Sj}, \mathrm{p}(\Theta \mathrm{b}+\tau)$ and $\Theta b 1$, with assumption that the Brown-Mayer's method of the second order is an optimal method of forecasting, are calculated according to expressions:

where:

$$
\mathrm{Sj}, \mathrm{p}(\Theta \mathrm{b}+\tau)=\mathrm{at}(0)+\tau \operatorname{at}(1)+\tau 2 \mathrm{at}(2)
$$

$$
\begin{gathered}
a_{t}^{(0)}=M_{t}=3\left(M_{t}^{(1)}-M_{t}^{(2)}\right)+M_{t}^{(3)} \\
a_{t}^{(1)}=T_{t}=\frac{\alpha}{2(1-\alpha)^{2}}\left\{(6-5 \alpha) M_{t}^{(1)}-2(5-4 \alpha) M_{t}^{(2)}+(4-3 \alpha) M_{t}(3)\right\} \\
a_{t}^{(2)}=Q_{t}=\frac{\alpha^{2}}{2(1-\alpha)^{2}}\left\{M_{t}^{(1)}-2 M_{t}^{(2)}+M_{\mathrm{t}}^{(3)}\right\} \\
M_{t}^{(1)}=\alpha \mathrm{y}_{\mathrm{t}}+(1-\alpha) M_{t-1}^{(1)} \\
M_{t}^{(2)}=\alpha M_{t}^{(1)}+(1-\alpha) M_{t-1}^{(2)} \\
M_{t}^{(3)}=\alpha M_{t}^{(2)}+(1-\alpha) M_{t-1}^{(3)}
\end{gathered}
$$

$\alpha$ - parameter of exponential smoothing, $\alpha \in(0,1)$

$$
\Theta_{b 1}=\Theta_{b}+\frac{\tau\left(S_{j, g}^{* *}-S_{j}\left(\Theta_{\mathrm{b}}\right)\right)}{S_{j}\left(\Theta_{b}+t\right)-S_{j}\left(\Theta_{b}\right)}
$$




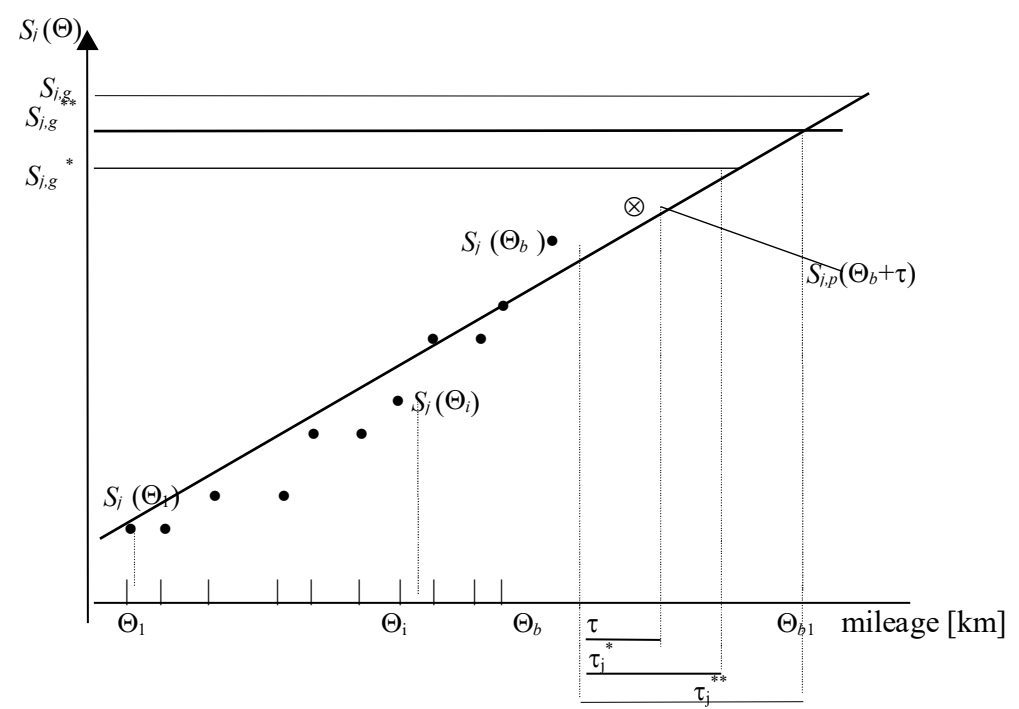

Fig.3. Determination of the term of the next devices diagnostication $\Theta_{b 1}$ by means of the method of levelling of diagnostic parameter limiting value for $\mathrm{S}_{\mathrm{j}}\left(\Theta_{\mathrm{b}}\right)>\mathrm{S}_{\mathrm{j}, \mathrm{gr}}\left[\Theta_{1}\right.$ - the beginning of device operation, $\Theta_{b}$ - the last device diagnostication, $\Theta_{b 1}$ - the term of the next device diagnostication].

Three options are then distinguished (see Fig.3):

a) not exceeding by a controlled diagnostic parameter a limit determined by a value $S_{j, g r^{*}}$ is interpreted as a lack of alarm signal for careful and more exact diagnostic observation of the device;

b) exceeding by a controlled diagnostic parameter a limit determined by a value $S_{j, g r *}$ is interpreted as an alarm signal for careful and more exact diagnostic observation of the device (alert threshold);

c) moment of exceeding by a controlled diagnostic parameter a limit determined by a value $S_{\mathrm{j}, \mathrm{gr} *}$ is interpreted as the time $\Theta_{\mathrm{b} 1}$ - i.e. the term of the next device diagnostication (alarm threshold).

\section{Method of determination of the time of reliable operation by means of estimation of diagnostic parameter change}

Increase of intensity of damages occurring as machine's operational potential wears implies a need of optimisation of diagnostication periodicity. From the course of intensity of machine's damages, it follows that within period of this intensity growth, the frequency of diagnostication should be increased. This is conductive to fall expenditures for machine's operation (decrease of: wears intensity, fuel consumption, spare parts wear, operational machines consumption etc.) and simultaneously costs and labour demand increase, as well as the time of excluding machine out of operation extends.

There are few possibilities of determination of diagnostication periodicity (the method of limiting symptoms values, the method of the smallest total operating costs), whilst for realisation of them numerous statistical data are indispensable, often inconvenient (in sense of amount and veracity) for obtaining.

In this point a problem of diagnostication periodicity has been considered in depiction of symptomatic reliability, taking advantage of the known symptom's limiting value [4]. 
Making $\mathrm{n}$ - measurements of a signal measure, chosen in separate procedure, it is possible to determine, based on it, the limiting value, depending on the relationship:

$$
\begin{gathered}
S_{j g r}=-\sigma_{s} \sqrt{\frac{P_{g}}{2 A}},[\text { Pg }- \text { repairing policy of the Works }] \\
\mathrm{A}=\mathrm{k}(1-\mathrm{Pg}), \mathrm{k}=\{1-9\}]
\end{gathered}
$$

and then determine the term of the next diagnostication $\Theta_{b 1}$ according to above proposed procedure.

Probability Pr of failure-free machine's work is determined by the ratio of number of machines Nk without damages with $\mathrm{S}_{\mathrm{j}}<\mathrm{S}_{\mathrm{jgr}}$ within inter-inspections period to machines' number $\mathrm{Np}$ based on considered [period of work. The number of machines with damages $\left(\mathrm{S}_{\mathrm{j}}\right.$ $>\mathrm{S}_{\mathrm{jgr}}$ ) $\mathrm{W}$ in assigned period of time amounts:

$$
\begin{array}{cc}
n=N_{p}-N_{k} \\
\text { and adequately: } & P_{r}=1-n / N_{p}
\end{array}
$$

The number of machines revealing $n$-failures within inter-revisions period, in relation to number of Sjgr exceeding amounts

$$
\mathrm{n}=\mathrm{N}_{\mathrm{s} r}\left[\mathrm{~S}\left(\theta_{\mathrm{i}}\right) \Theta_{\mathrm{b} 1}\right]
$$

where: $\mathrm{N}_{\mathrm{sr}}=\left(\mathrm{N}_{\mathrm{p}}+\mathrm{N}_{\mathrm{k}}\right) / 2 ; \Theta_{\mathrm{b} 1}$ - time of the next diagnostication $(\mathrm{km}$, hour); $\mathrm{S}(\theta \mathrm{i})$ - parameter characterising an ascending dynamic for symptomatic curve of life in considered period $\Theta_{\mathrm{j}}$, defined according to relationship:

$$
S\left(\Theta_{i}\right)=\left(\frac{S_{j}}{S_{j g r}-S_{j}}\right)\left(\frac{1}{\Theta_{j}}\right)[1 / \mathrm{km}, \operatorname{god} z]
$$

where: $\mathrm{S}_{\mathrm{j}}$ - value of measured symptom, $\mathrm{S}_{\mathrm{jgr}}$ - limiting value of the symptom.

Providing (13) to (12) after transformation we obtain following relationships:

$$
\begin{aligned}
P_{r} & =\frac{2-S\left(\Theta_{i}\right) \cdot \Theta_{b 1}}{2+S\left(\Theta_{i}\right) \cdot \Theta_{b 1}} \\
\Theta_{b 1} & =\frac{2\left(1-P_{r}\right)}{S\left(\Theta_{i}\right)\left(1+P_{r}\right)}
\end{aligned}
$$

With exponential syndrome distribution in time $\Theta_{\mathrm{j}}$ the above equations assume following form:

$$
\begin{aligned}
& P_{r}=e^{-S\left(\Theta_{i}\right) \Theta_{b 1}} \\
& \Theta_{b 1}=\frac{-\ln P_{r}}{S\left(\Theta_{i}\right)}
\end{aligned}
$$

Assuming $1<\mathrm{P}_{\mathrm{r}}<0,8$ with an accuracy sufficient for practice one may use an approximate expression:

$$
\mathrm{Pr}_{\mathrm{r}}=1-\mathrm{S}(\theta \mathrm{i}) \Theta_{\mathrm{b} 1}
$$

Assuming constant intensity of symptomatic curve of life ascending (assigned Pr) we achieve:

$$
\mathrm{P}_{\mathrm{r}}=\text { const } \quad \mathrm{S}(\theta \mathrm{i}) \Theta_{\mathrm{b} 1}=\text { const }
$$

From which it results that the higher $\mathrm{S}(\theta \mathrm{i})$ the smaller $\Theta b 1$, what in interpretation of symptomatic diagnostics means, that as a symptom increase (approaching to Sjgr) within machine's time-life $\Theta \mathrm{j}$, diagnostication frequency increases (fig.4). 


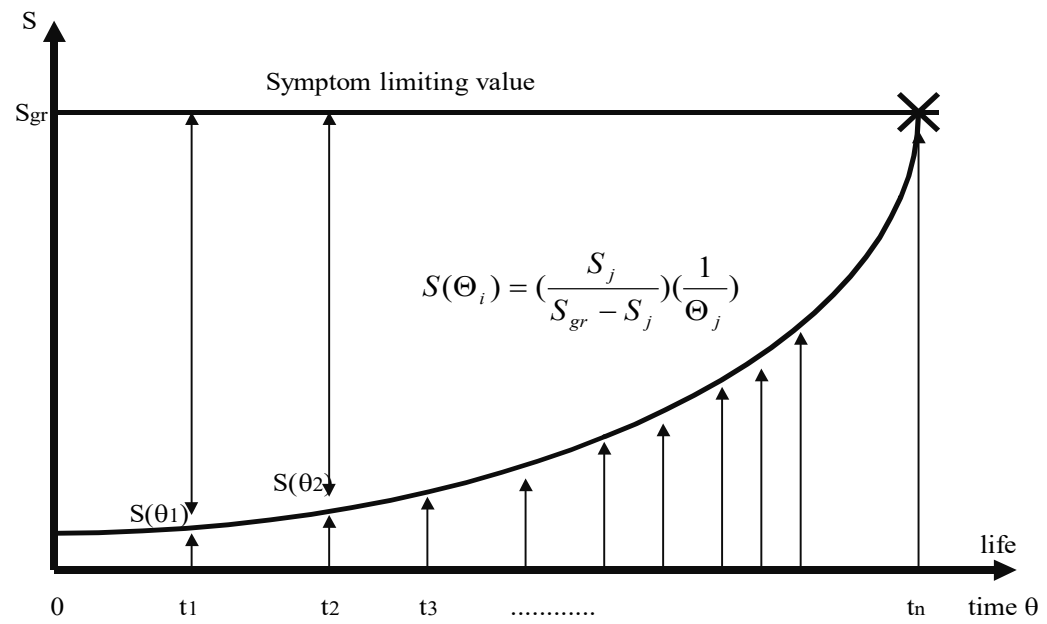

Fig.4. Periodicity of diagnostication in symptomatic depiction.

From the relationship (17) one may thus determine $\Theta_{\mathrm{b} 1}$ :

finally:

$$
\Theta_{b 1}=\frac{1-P_{r}}{S\left(\Theta_{i}\right)} \text { and after providing a relationship }
$$

$$
\Theta_{b 1}=\frac{\left(1-P_{r}\right)\left(S_{g r}-S_{j}\right)}{S_{j}} \Theta \mathrm{j}
$$

Presented relationship allows to determine the term of the next diagnostication in relation to current value of measured symptom, its relation to symptom's limiting value and with considering the repairing policy of the works $[3,4]$.

\section{Conclusions}

In this elaboration there are presented three methods of determination of devices reliable operation time in form of the term of the next technical equipment diagnostication for needs of strategy of operation based on technical state:

a) by means of forecast error value levelling,

b) by means of levelling of diagnostic parameter limiting value,

c) by means of diagnostic parameter change estimation.

Carried out verifying examinations of efficiency of proposed methods reveal some limitations and preferences in their practical application.

\section{Bibliography}

1. H.Tylicki: Method of determination of terms of mechanical vehicles units' operations. Scientific Notebooks Problems of Operation no. 5/97, ITE Radom 1997, p. 437-449.

2. H.Tylicki: Optimisation of process of forecasting of mechanical vehicles technical state. University Publishers of University of Technology and Agriculture, Bydgoszcz 1997, p. 93-129.

3. B.Żółtowski: Conditions of states classification in machines diagnostics. Scientific Notebooks Problems of Operation no. 4/97, ITE Radom 1997, p. 37-57. 
4. B.Żółtowski: The basics of machines diagnostics. University Publishers of University of Technology and Agriculture, Bydgoszcz, 1996.

DATA ABOUT AUTHOR:

Professor Bogdan ŻÓŁTOWSKI, engineer 